\title{
Early Complications from Absorbable Anchoring Suture Following Thread-Lift for Facial Rejuvenation
}

Seung Hun Yeo ${ }^{1}$, Young Bae Lee ${ }^{2}$, Dong Gil Han ${ }^{1}$

${ }^{1}$ Department of Plastic and Reconstructive Surgery, Catholic University of Daegu, School of Medicine, Daegu; ${ }^{2}$ LIFT Plastic Clinic, Daegu, Korea

This article was presented as a free paper at the 74th congress of the Korean Society of Plastic and Reconstructive Surgeons on November 19, 2016, in Seoul, Korea.

No potential conflict of interest relevant to this article was reported.
Background Thread lifting has been known as a minimally invasive procedure compared with the standard incisional surgery for facial rejuvenation. Although there have been several reports on acute or delayed complications after using nonabsorbable thread type, there have only been a few studies on such complications after using absorbable thread type. Hence, the aim of this study was to introduce various complications of thread lifting using the absorbable anchoring type for facial rejuvenation and to discuss about how to resolve it.

Methods A retrospective review of 144 cosmetic patients, who underwent absorbable barbed thread lifting between July 2013 and December 2015, was performed. The procedure was done using the temporal anchoring technique. The thread used in this study are polydioxanone molding $\operatorname{cog}(\mathrm{PMC})$ and polydioxanone cutting $\operatorname{cog}(\mathrm{PCC})$, which are both the absorbable type.

Results The results are as follows: Dimple was developed in 3 cases (2.1\%); thread exposure was developed in 5 cases (3.5\%); alopecia was developed in 3 cases (2.1\%); undercorrection was developed in 3 cases (2.1\%); asymmetry was developed in 1 case (0.7\%); and parotid gland injury was developed in 1 case (0.7\%). Out of the total 144 patients, sixteen (11.1\%) patients developed at least 1 complication. There was no detectable inflammation or consistent skin excavation.

Conclusions Most complications of absorbable thread lifting are minor ones, and such complications resolve by retouching or widening the anchoring gap, but it is important to keep in mind the possibility of parotid gland injury.

Keywords Rejuvenation, Rhytidoplasty, Suture anchors

\section{INTRODUCTION}

Thread lifting has been known as a minimally invasive procedure with limited scarring, rapid recovery, and minimal complications compared with the standard incisional surgery for facial rejuvenation $[1,2]$.

Received: Dec 21, 2016 Revised: Jan 17, 2017 Accepted: Jan 23, 2017 Correspondence: Dong Gil Han Department of Plastic and Reconstructive Surgery, Daegu Catholic University Medical Center, Catholic University of Daegu School of Medicine, 33 Duryugongwon-ro 17-gil, Nam-gu, Daegu 42472, Korea. E-mail: dghan1001@cu.ac.kr

Copyright @ 2017 The Korean Society for Aesthetic Plastic Surgery.

This is an Open Access article distributed under the terms of the Creative Commons Attribution Non-Commercial License (http://creativecommons.org/licenses/by-nc/4.0/) which permits unrestricted non-commercial use, distribution, and reproduction in any medium, provided the original work is properly cited. $\quad$ www.e-aaps.org
Thread lifting began in the late 1990s by Dr. Sulamanidze, who introduced the Aptos threads [3]. After receiving an approval from the U. S. Food and Drug Administration (FDA) in 2005, thread lifting began to grow in popularity, and a variety of techniques have been developed since then, such as Woffels thread lifting, Waptos suture lifting, Isse unidirectional barbed thread lifting, and silhouette lifting $[4,5]$. In Korea, an absorbable thread began to be used to raise the skin elasticity, and it was applied to thread lifting [6].

Although several acute or delayed complications after using nonabsorbable thread type were reported [5,7-10], few studies on the absorbable thread type have examined its associated risks and complications. Moreover, the majority of previous studies reported only about the floating type, ignoring the anchoring type.

Therefore, the aim of this study was to introduce the various complications of thread lifting using the absorbable anchoring type for facial rejuvenation and to discuss about how to avoid it. 


\section{METHODS}

This was a retrospective review of 144 patients, who underwent absorbable barbed thread lifting on cheek bilaterally for cosmetic purpose between July 2013 and December 2015. The follow-up period of patients was 0 to 52 weeks. Patients with good skin elasticity and less lower facial fat underwent thread lifting only. Others with moderate-to-poor skin elasticity and a much lower facial fat underwent thread lifting with liposuction of the lower face. Liposuction was done on both cheek or chin area using $3 \mathrm{~mm}$ cannula.

\section{Surgical technique}

The procedure was performed using the temporal anchoring type, and the number of threads placed appropriately according to the
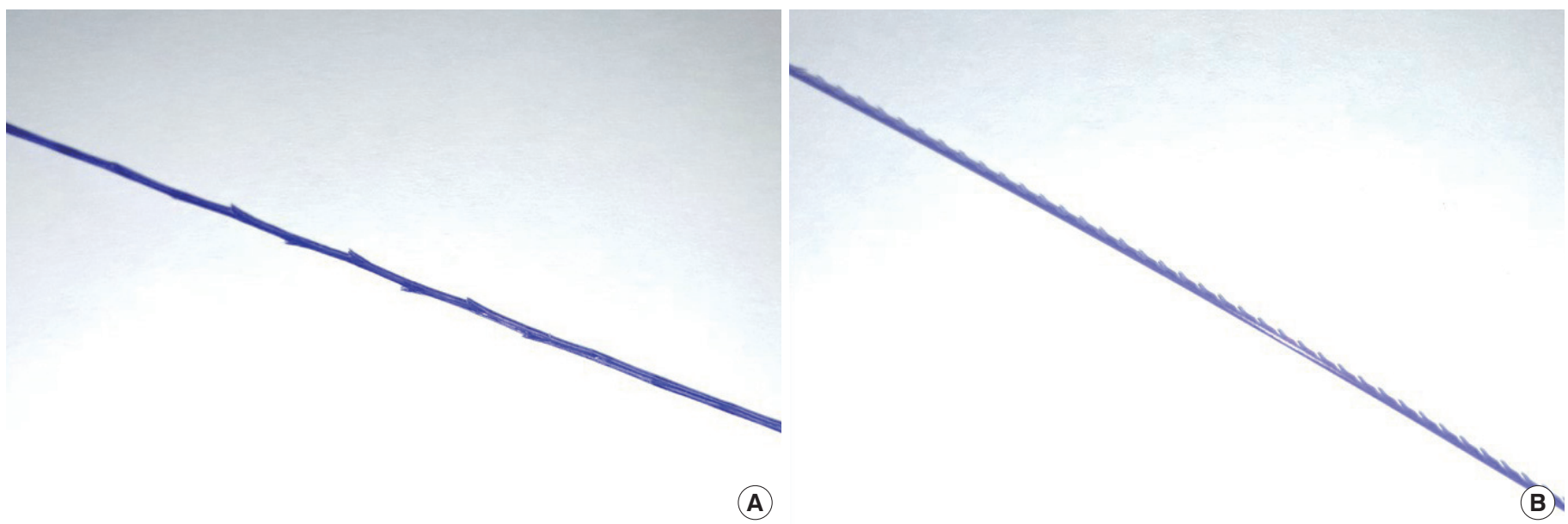

Fig. 1. Thread type. (A) MINT Lift ${ }^{\circledR}$ (minimal invasive non-surgical thread; HansBiomed, Seoul, Korea), Polydioxanone (PDO) molding cog. (B) Omega $41^{\circledR}$ (OV World, Seoul, Korea), PDO cutting cog.
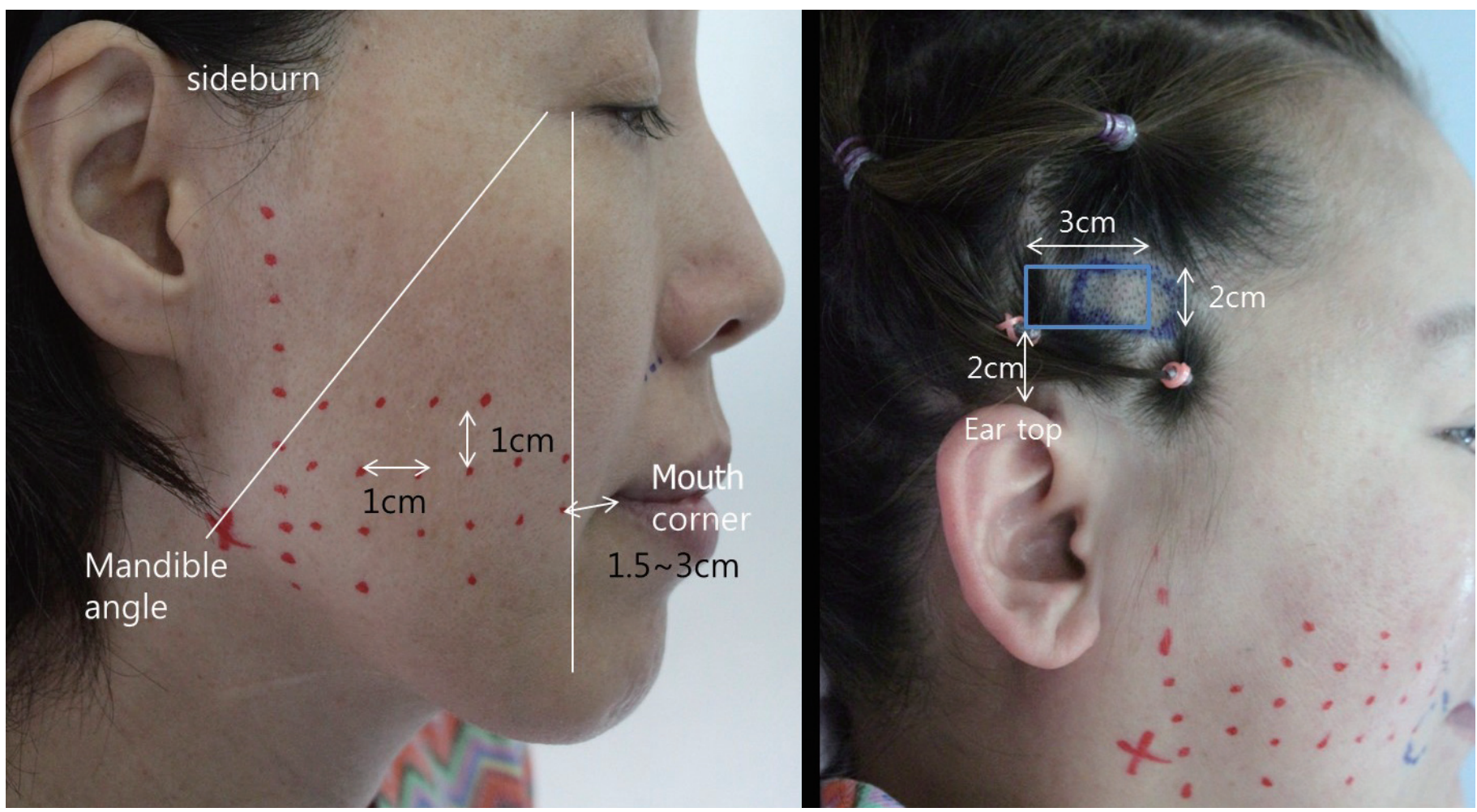

Fig. 2. Design for absorbable thread lift. Design conducted at least 2 to $3 \mathrm{~cm}$ from the mouth corner, and the distance between the thread was about $1 \mathrm{~cm}$. Shaving was done on the temporal anchoring site about $3 \times 2 \mathrm{~cm}$. 
facial regions which be treated. The thread used in this study was MINT Lift ${ }^{\circledast}$ ML-1043 (minimal invasive non-surgical thread; HansBiomed, Seoul, Korea) and omega $41^{\circledR}$ (OV World, Seoul, Korea) which are biodegradable polydioxanone thread (Fig. 1).

The design was conducted at least 2 to $3 \mathrm{~cm}$ from the mouth corner. The distance between the threads was about $1 \mathrm{~cm}$. We drew two virtual lines; one from the lateral canthus to the mandibular angle, and another as a vertical line from the lateral canthus. The thread exit point area was located between these two lines. We defined an area $2 \mathrm{~cm}$ superior to the top of the ear as the anchoring site and shaved an area of about $3 \times 2 \mathrm{~cm}$ (Fig. 2). The curved needle with threads entered the temporal anchoring site, and the threads were anchored in the deep temporal fascia. A long needle absorbable threaded was inserted into the hole and advanced through the beneath subcutaneous layer, and penetrated skin on designed exit point. After repeating this step, excess threads that came out from the exit point were cut and gently pulled out. Moreover, the thread was slightly pushed to get it buried under the skin. After performing the procedure, the dimple effects were removed by manual pressure [11].

The thread lifts were performed under local anesthesia using 2.0 $\mathrm{mL}$ of $2 \%$ lidocaine with 1:100,000 epinephrine and tumescent 10 cc of normal saline per side hemi-face around thread pathway area.

\section{RESULTS}

One hundred and forty-four women underwent thread lifting using the barbed, unidirectional, absorbable sutures. The average follow-up period was 11.1 weeks, and the average age of these women was 42.5-year-old (Table 1).

A total of 951 threads were placed in these 144 patients with a subcutaneous location. On average, 6.6 threads were placed in each patient. The thread lift alone was employed in 42 of the 144 patients (29.2\%), whereas additional procedures were performed simultaneously in the remaining 102 patients (70.8\%). Pain, numbness, discomfort and mild bleeding were excluded in this report, as most patients suffered them and these were transient conditions and recovered by itself within one month [1]. Dimple, thread exposure, alopecia, undercorrection, asymmetry and parotid gland injury were considered here as major complications, as these were longterm impact that required additional procedures. Sixteen of the 144 patients (11.1\%) developed at least one complication (Table 2).

Table 1. Demographics of patients

\begin{tabular}{lc}
\hline Characteristics & Mean (range) \\
\hline Age (year) & $42.5(21-65)$ \\
Thread number & $6.6(2-14)$ \\
Follow-up (week) & $11.1(0-52)$ \\
\hline
\end{tabular}

There was no detectable inflammation or consistent skin excavation.

\section{DISCUSSION}

Nowadays, patients prefer simple procedures, early recovery, and immediate outcome. As a result, the interest in face lifting using a thread has drastically increased recently. Accordingly, research and development for a more efficient and effective surgical method are being performed. At present, many kinds of threads (e.g., omega 41, MINT, Blue rose) have been developed according to manufacturers, components, and difference among synthetic methods of $\operatorname{cog}$, and widely used. However, to date, there have not been any studies on absorbable thread lifts, despite many studies on non-absorbable thread lifts. So we aim this point, and studied about complications of the absorbable thread lift for facial rejuvenation.

In our study, twelve patients out of the 144 patients developed complications, including dimple, thread exposure, alopecia and parotid gland injury.

Dimple has developed in 3 cases (2.1\%). In a 43-year-old female patient, dimple has developed at 5 days after thread lifting, which became clear as swelling decreased. It has disappeared by lightly retouching the area from top to bottom. In another case, dimple has developed at 3 weeks after thread lifting in a 23 -year-old female patient (Fig. 3) with history of zygoma reduction and liposuction on both cheeks 2 years ago. The dimple has not disappeared after manual reduction, so a cannula dissection was performed; however, it did not improve and it has barely disappeared at 3 months after lifting. It is thought that dimples have not been easily corrected due to inner scarring from the previous procedure. If the depth of the advancing thread is much more superficial or not uniform, a depression will be occurred at a portion located closely to the skin, and which can be dimple or irregular contour. Most cases of dimples were almost resolved spontaneously. But remarkable dimples were managed by manual reduction and that time, sometimes the effect of thread lift was reduced when remove dimple completely.

Table 2. Absorbable thread complication rate

\begin{tabular}{lccc}
\hline & \multicolumn{2}{c}{ Absorbable thread type } & \\
\cline { 2 - 3 } Complications & $\begin{array}{c}\text { PDo molding cog } \\
\text { (n = 88) }\end{array}$ & $\begin{array}{c}\text { PDo cutting cog } \\
\text { (n = 56) }\end{array}$ & $\mathrm{n}(\%)$ \\
\hline Dimple & 1 & 2 & $3(2.1)$ \\
Thread exposure & 3 & 2 & $5(3.5)$ \\
Alopecia & 3 & 0 & $3(2.1)$ \\
Undercorrection & 0 & 3 & $3(2.1)$ \\
Asymmetry & 0 & 1 & $1(0.7)$ \\
Parotid gland injury & 0 & 1 & $1(0.7)$ \\
Total & 7 & 9 & $16(11.1)$ \\
\hline
\end{tabular}

PDO, polydioxanone. 

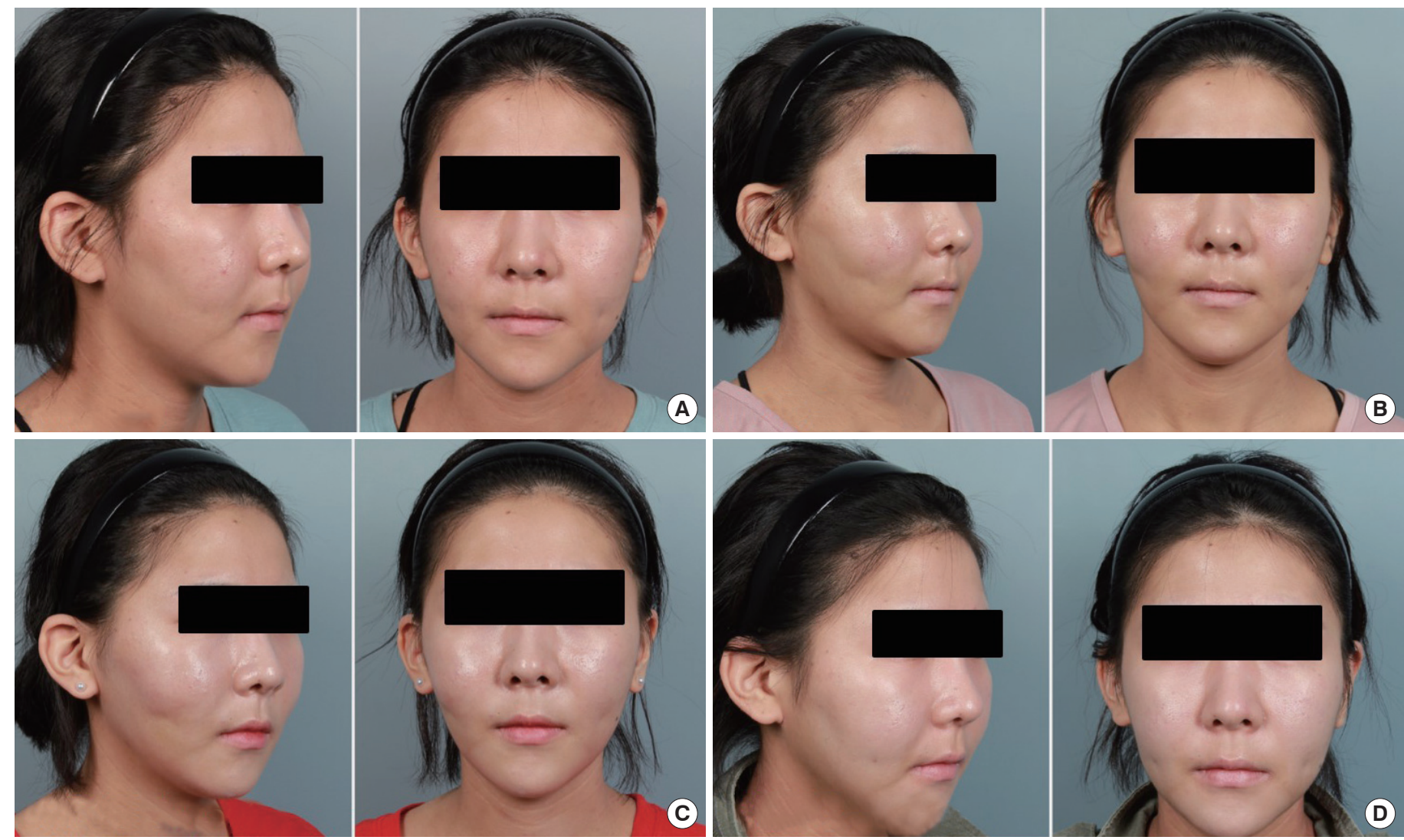

Fig. 3. Dimple case. (A) A 23-year-old patient is shown before procedure. (B) The patient had a dimple on the right cheek 3 weeks after the procedure. She had a history of zygoma reduction and liposuction on both cheeks 2 years ago. (C) Persistent of dimple on right cheek 1 month after. (D) Dimple disappeared at 3 months after.
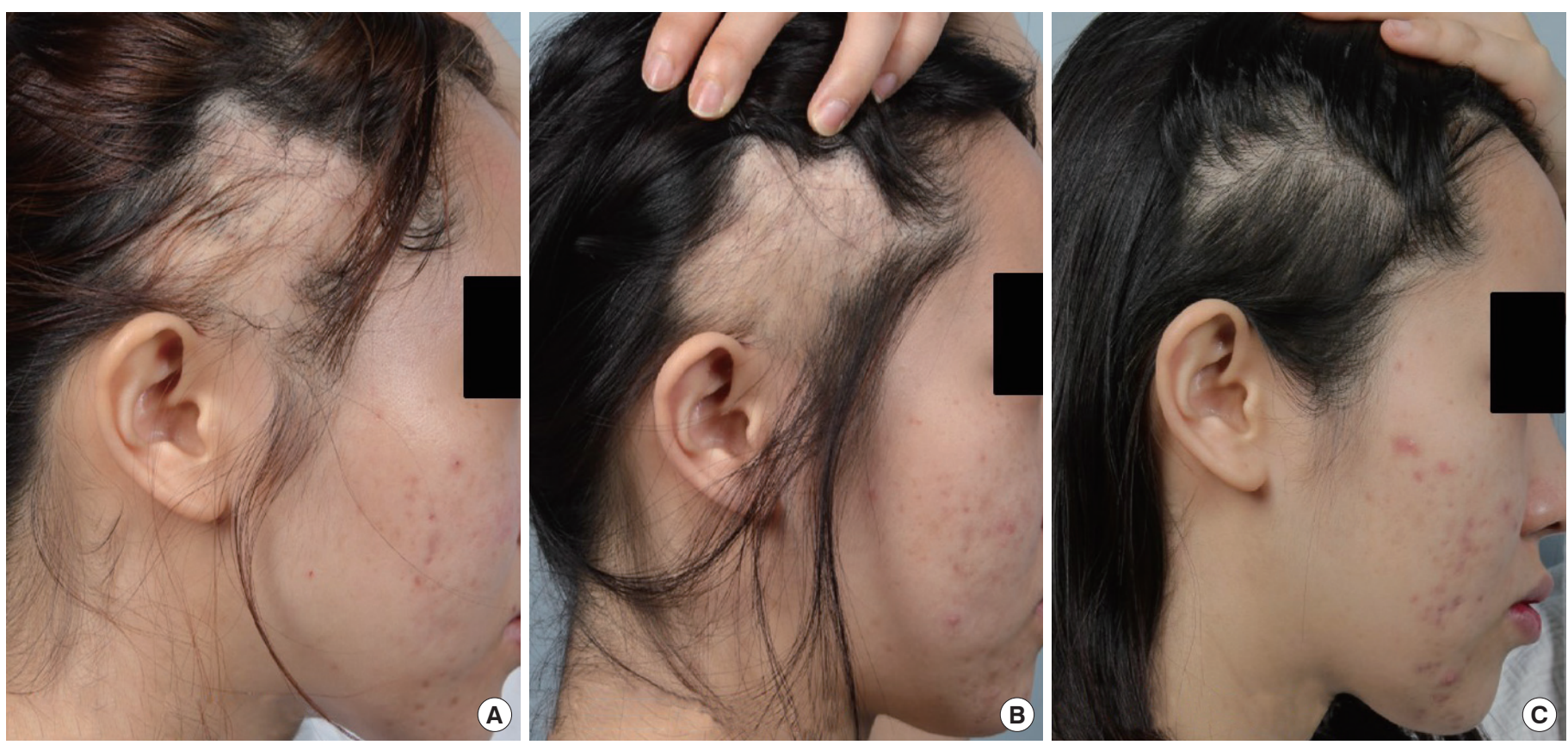

Fig. 4. Alopecia case. (A) A 23-year-old patient had alopecia on the right temple 3 weeks after the procedure. (B) It progressed more until 2 months. (C) Alopecia was resolved at 5 months by itself. 
So after a couple of weeks, dimple was removed.

Thread exposure developed in 5 cases (3.5\%), and it was the most common complication. In 2 cases, the thread was removed because it was palpable on the left cheek at 1 month after thread lifting. In the other 3 cases, it was removed at 2 months. Thread exposure is caused by migration of thread to superficial layer and repeated inflammation reaction. Even though remove exposed thread, there is no remarkable change of thread lift effect, because exposed thread is only a part of it and it occurred at least 2 month after procedure.

Alopecia has developed in 3 cases (2.1\%). Among these cases, a 23-year-old female patient (Fig. 4) showed alopecia at 3 weeks after thread lifting, which further progressed until 2 months after lifting and was resolved at 5 months. All 3 cases of hair loss occurred after shaving smaller portions, and did not occur after widening the anchoring gap. Ischemia due to tension in the anchoring process is thought to be the cause of alopecia.

Undercorrection has developed in 3 case (2.1\%) and asymmetry has developed in 1 case (0.7\%). Undercorrection has developed in 2 cases at the first month after thread lifting and in 1 case at 6 months. Among these cases, one patient wanted additional thread lift. Asymmetry has developed in a 40-year old female patient and she visited because of right cheek sagging symptom at 4 months after thread lifting. The latter's thread lift effect is better than the former. When performing additional thread lift, it must be done after swelling has subsided. Also it has been observed that undercorrection and asymmetry developed only when polydioxanone cutting $\operatorname{cog}$ (PCC) is used.

Parotid gland injury has developed in 1 case $(0.7 \%)$. Intermittent swelling and inflammation occurred at 1 month after the thread lifting in a 50-year-old female patient (Fig. 5). Antibiotics and antiinflammatory medication was prescribed under the impression of inflammation by the thread. Just after taking a dose, symptoms were relieved but they recurred. Ultrasonography was done at 3 months, and it showed that the thread passes through the parotid gland. After observation with conservative treatment, the condition had improved after 5 months.

Injury to the parotid gland and duct is one of the most dangerous complications during the thread lifting [12]. Anatomically, procedures should be done carefully at the inferior side of the parotid gland and from the posterior side of the masseter to the mandible angle because they are tightly attached to superficial facial fascia (SMAS) and parotidomasseteric fascia [13]. Moreover, according to recent articles, the pathway of the parotid gland is more accurate as the lower line connecting the tragus and mouth corner than as
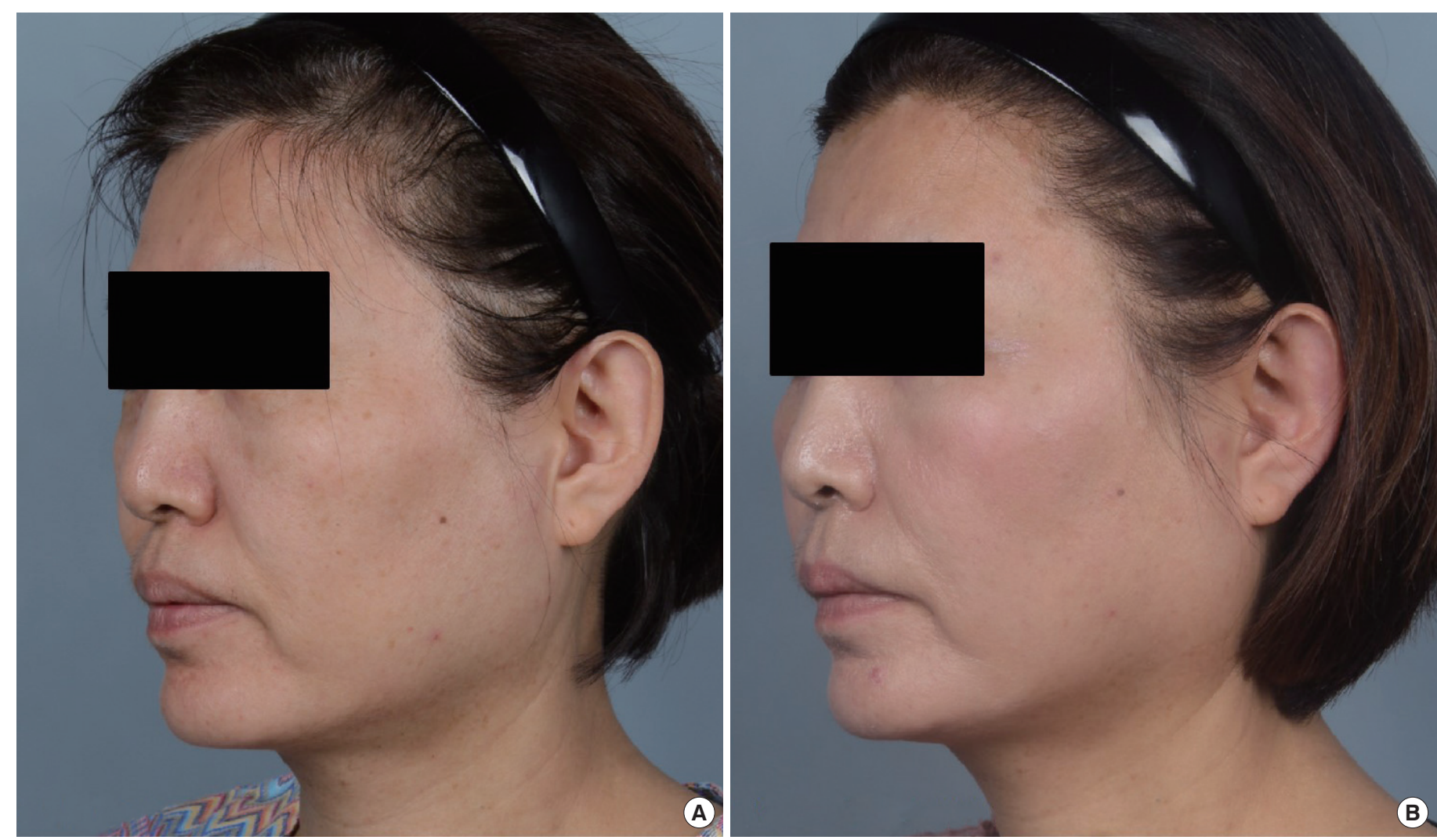

Fig. 5. Parotid gland injury. (A) A 50-year-old patient is shown before the procedure. (B) At 1 month after the thread lifting, intermittent swelling occurred and signs of infection showed. Ultrasonography showed the thread passing through the parotid gland. After observation with antibiotics, patient's condition improved after 5 months. 
the existing concept of the line connecting the tragus and mid-philtrum [14]. To avoid these serious complications, it is important to recognize the anatomical structure and perform thread lift along precise layers not to be too deep.

In conclusion, most complications of the absorbable thread lifting are minor, but it is important to keep in mind the possibility of parotid gland injury.

This study has a limitation when interpreting whether absorbable thread is safer than non-absorbable thread because most complications have developed within a 6-month period. However, it is worth noting that the absorption period of absorbable thread is usually 6 to 8 months [6]. Thus further studies needed with long term follow-ups.

\section{PATIENT CONSENT}

Patients provided written consent for the use of their images.

\section{REFERENCES}

1. Sulamanidze M, Sulamanidze G, Vozdvizhensky I, et al. Avoiding complications with Aptos sutures. Aesthet Surg J 2011;31:863-73.

2. Kaminer MS, Mandy S. ContourLift ${ }^{\text {tix }}$ : A new method of minimally invasive facial rejuvenation. Cosmet Dermatol 2007;20:29-35.

3. Sulamanidze MA, Fournier PF, Paikidze TG, et al. Removal of facial soft tissue ptosis with special threads. Dermatol Surg 2002;28:367-71.

4. Paul MD. Barbed sutures in aesthetic plastic surgery: evolution of thought and process. Aesthet Surg J 2013;33:17s-31s.
5. Rachel JD, Lack EB, Larson B. Incidence of complications and early recurrence in 29 patients after facial rejuvenation with barbed suture lifting. Dermatol Surg 2010;36:348-54.

6. The Korean Society of Aesthetic Plastic Surgery. Aesthetic plastic surgery. Paju: Koonja; 2016.

7. Garvey PB, Ricciardelli EJ, Gampper T. Outcomes in threadlift for facial rejuvenation. Ann Plast Surg 2009;62:482-5.

8. Yoo KH, Kim WS, Hong CK, et al. Chronic inflammatory reaction after thread lifting: delayed unusual complication of nonabsorbable thread. Dermatol Surg 2015;41:510-3.

9. Barron R, Margulis A, Icekson M, et al. Iatrogenic parotid sialocele following rhytidectomy: diagnosis and treatment. Plast Reconstr Surg 2001;108:1782-4; discussion 5-6.

10. Suh DH, Jang HW, Lee SJ, et al. Outcomes of polydioxanone knotless thread lifting for facial rejuvenation. Dermatol Surg 2015;41:720-5.

11. Abraham RF, DeFatta RJ, Williams EF, 3rd. Thread-lift for facial rejuvenation: assessment of long-term results. Arch Facial Plast Surg 2009; 11:178-83.

12. Winkler E, Goldan O, Regev E, et al. Stensen duct rupture (sialocele) and other complications of the Aptos thread technique. Plast Reconstr Surg 2006;118:1468-71.

13. Lawson GA, 3rd, Kreymerman P, Nahai F. An unusual complication following rhytidectomy: iatrogenic parotid injury resulting in parotid fistula/sialocele. Aesthet Surg J 2012;32:814-21.

14. Stringer MD, Mirjalili SA, Meredith SJ, et al. Redefining the surface anatomy of the parotid duct: an in vivo ultrasound study. Plast Reconstr Surg 2012;130:1032-7. 